\title{
In vitro implant-bone interface pressure measurements for a cementless femoral implant. A preliminary study
}

\author{
Aurélien Courvoisier ${ }^{\mathrm{a},{ }^{*}}$, Valéry Barbour ${ }^{\mathrm{b}}$, Wafa Skalli ${ }^{\mathrm{b}}$, David Mitton ${ }^{\mathrm{c}}$ \\ ${ }^{a}$ Pediatric Orthopedic Department, Grenoble University Hospital, Grenoble Alpes University, TIMC IMAG - SPM, BP 217, 38043 Grenoble Cedex 09, France \\ ${ }^{\mathrm{b}}$ Institut de Biomécanique Humaine Georges Charpak, Arts et Métiers ParisTech, 151 Bvd de l'hôpital, 75013 Paris, France \\ ${ }^{\mathrm{c}}$ Laboratoire de Biomécanique et Mécanique des Chocs UMR_T 9406 (IFSTTAR-UCBL), 25 Avenue François Mitterrand, Cité des Mobilités, 69675 Bron, \\ France
}

\begin{abstract}
A B S T R A C T
Purpose: Implants endurance as well as a good clinical tolerance depends on the recovery of a physiological stress distribution within bone after implantation. The purpose of the present work was to develop an alternative technique using Force Sensing Resistors (FSR) to gather in vitro pressure values at the implant-bone interface for a cementless implant.

Method: Eight cementless femoral stems were instrumented with six calibrated FSR bonded on each facet and then implanted in eight cadaver femurs. Compression tests were performed until failure and FSR pressure values were recorded.

Results: The average failure load was $4241 \mathrm{~N}$. The maximum contact pressure measured with the FSR averaged $1.965 \mathrm{MPa}$.

Conclusion: FSR reached many of the requirements for an ideal implant-bone interfacial sensor. This experimentation provided in vitro quantitative data on contact pressure at the implant-bone interface, which could help understanding stress shielding phenomenon and developing relevant numerical model.
\end{abstract}

\section{Introduction}

Load transfer is a major issue in joint replacement biomechanics. Implants endurance as well as a good clinical tolerance depends on the recovery of a physiological stress distribution within bone after implantation. Bone remodeling of the implanted femur depends on the stress distribution within the femur [1] which relies on the implant primary stability and the implant-bone load transfer. Each part of this osteointegration process has been previously investigated using in vitro and numeric experimentations [2-5]. Additionally, an abnormal stress distribution within the implant-bone interface tends to generate stress shielding, which is one of the main causes of implants aseptic loosening and bone resorption [6]. To investigate this problem, most authors have used numerical models $[7,8]$ or in vitro experimentations $[9,10]$.

The main issue when investigating bone implant-bone load transfer is to collect measurements within the implant-bone interface. Repeatability and accuracy of such in vitro experimentations undergo technical limitations of the different types of transducers available which are commonly based on strain gauges. These transducers are too fragile to sustain implantation of a femoral implant unless embedded in the cement. Previously published works provide strain measurements on the external cortex of the femur and within the cement layer but none within the bone-implant interface for cementless implants [10-13]. Distribution of contact pressure for cementless implants has been investigated in vitro using pressure films and tactile sensors but in bisected synthetic femurs \{Sakai, 2006 \#9\}. But alteration of the femur structure is not suitable when dealing with pre-clinical validation of femoral components.

The purpose of the present work was to develop an alternative technique using Force Sensing Resistors (FSR) to gather in vitro pressure values at the implant-bone interface cementless implants.

\section{Materials and methods}

\subsection{Sensor selection}

Force Sensing Resistors (I.E.E. - FSR - 149) display a decrease in resistance with an increase in the normal force applied to the active 
surface. The smallest type of FSR able to fit each facet of the prosthesis was selected for this application. This sensor has a disc shaped active area with an internal diameter of $5 \mathrm{~mm}$ and an external diameter of $7.6 \mathrm{~mm}$. The total length of the device is of $38.1 \mathrm{~mm}$ and the nominal thickness of $0.3 \mathrm{~mm}$. The FSR is a thickfilm device consisting of two conducting interdigitated patterns deposited on a thermoplastic sheet facing another sheet containing a conductive polyetherimide film. A spacer placed between the plastic sheets permits the two sheets to make electrical contact when the force is applied.

The common range of force measurements is $0-100 \mathrm{~N}$ but the force sensitivity ranges from below $100 \mathrm{~g}$ (break force stands between $20 \mathrm{~g}$ and $100 \mathrm{~g}$ ) to over $10 \mathrm{~kg}$ and the force resolution is better than $0.5 \%$ full scale. For the specific FSR used in this study, the pressure sensitivity ranges from below $0.05 \mathrm{MPa}$ to over $5 \mathrm{MPa}$. The single part force repeatability is + or $-2 \%$ to $5 \%$ of established nominal resistance. The stand-off resistance is over $1 \mathrm{M}$ Ohms.

Each FSR was soldered to lead wires. The soldered area was then enfolded with a plastic adhesive to guarantee permanent electrical continuity.

\subsection{Sensor calibration}

Each FSR was calibrated twice for compressive load values ranging from 0 to $15 \mathrm{MPa}$ using a loading machine (5566, Instron, Canton, MA, USA) at a constant displacement rate of $7 \mathrm{~mm} / \mathrm{min}$. The calibration process was done using a custom program in Labview. A $16 \times 16 \mathrm{~mm}$ square of felt was used to actuate the FSR device (Fig. 1). A rolling of the felt actuator was performed before the tests to stabilize its behavior under compression. Load values were converted in pressure values (Psensor $=$ Pfelt $=$ Fmeasured/Sfelt). A multilinear regression was determined in LabView (National Instruments) linking pressure to the measured output voltage. A second calibration of the FSR was performed using the same process. The whole calibration process was restarted in case of divergence between the two curves obtained.

The repeatability of the calibration procedure was investigated on one FSR before the entire bench tests. Ten actuations were performed and the curves obtained may be superimposed (Fig. 2).

\subsection{Sensor fixation}

Six force sensing resistors (FSR) (I.E.E. - FSR - 149) were bonded to the femoral stem after local removing the HA coating and subsequently coated with plastic adhesive to guarantee

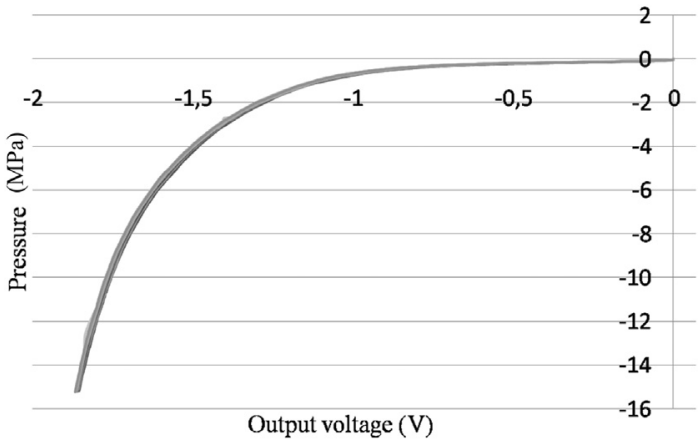

Fig. 2. Comparison of the responses of one FSR to ten consecutive actuations.

impermeability and permanent electrical continuity (Fig. 3). FSR location on the prosthesis was arbitrary selected but similar for each stem. The lead wires were laid in the grooves of the prosthesis. FSR 1, 2, 3 and 4 were bonded at the same level: FSR 1 on the anterior facet of the stem, FSR 2 symmetrically on the posterior facet, FSR 3 on the lateral facet and FSR 4 on the medial facet. FSR 5 was bonded on lateral facet proximally to FSR 3 and FSR 6 placed under the stem's collar (Fig. 3). A zero setting was performed for each FSR after fixation to eliminate pre-loading.

\subsection{Electrical interface}

A voltage divider was recommended for the use of FSR. This circuit produced an output voltage that was a fraction of its input voltage (Fig. 4). Voltage division referred to the partitioning of a voltage among the resistances (FSR and a $2 \mathrm{M} \Omega$ resistance). Since the FSR resistance was inversely proportional to applied force. The final result is a direct proportionality between force and voltage.

The output from the amplifier (Metrix AX 321, \pm 5 V DC) was fed into a custom developed circuit which was connected to a commercial 32-channel board (SCXI-1300, SCXI-1314, National Instruments, Austin, TX, USA) mounted on a personal computer. Multichannel data were acquired by a custom developed software, using LabView (National Instruments).

\subsection{Implantation of instrumented femoral stem}

Eight right femurs from two female and six male cadavers were harvested fresh and stored at $-20^{\circ} \mathrm{C}$. The mean age at death was 80.25 years [Range, 51-91] and the interval between death and

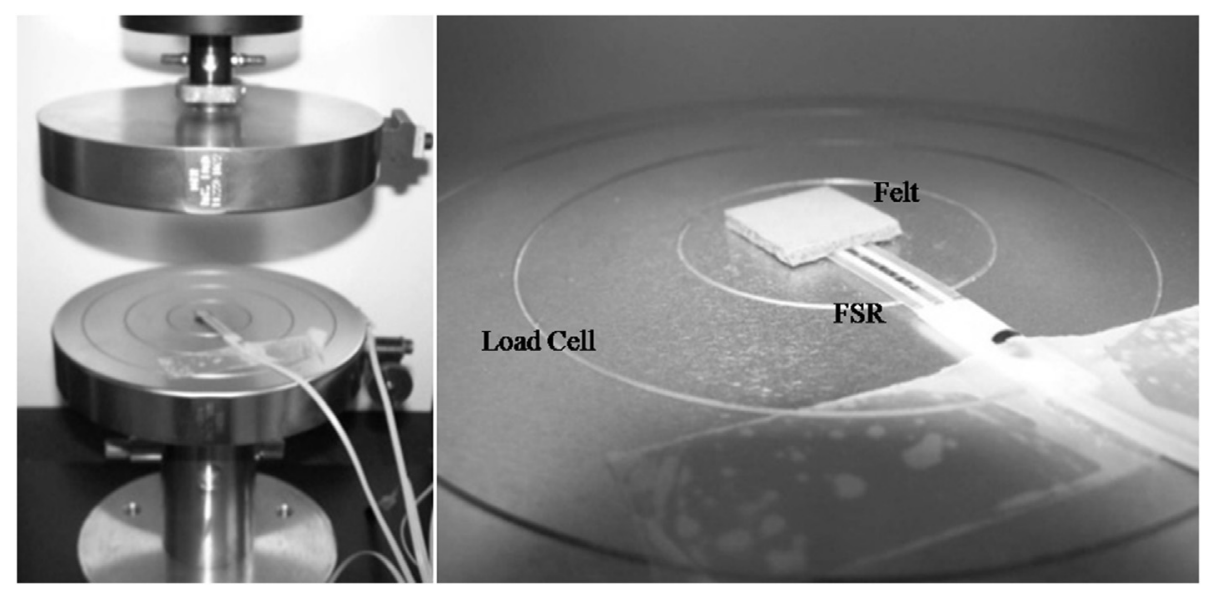

Fig. 1. Actuation system used for calibration of force sensing resistors (FSR). 


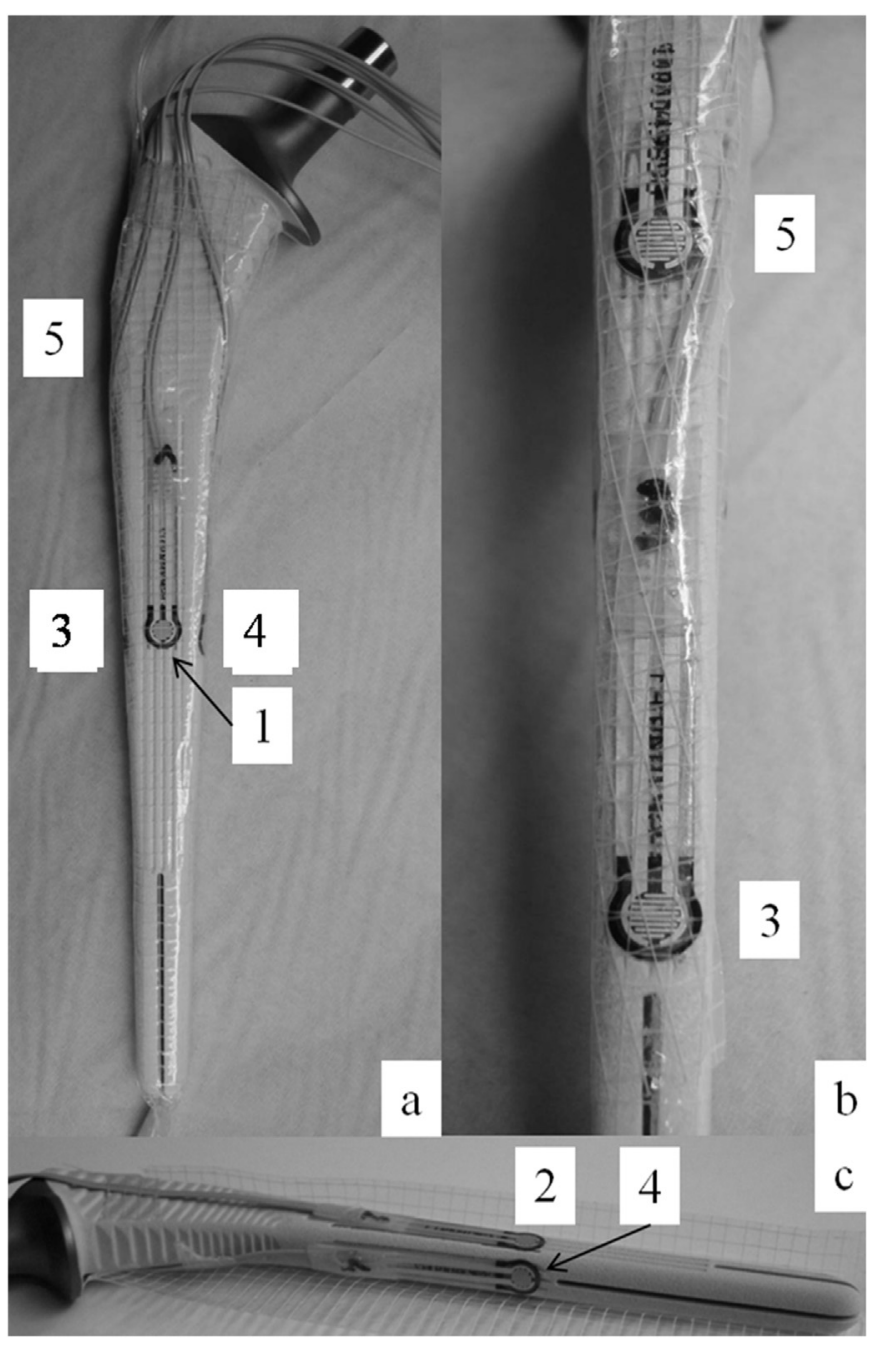

Fig. 3. Photographs showing the positioning of the FSR on the femoral stem. Six FSR were bonded to the femoral stem: FSR 1 on the anterior facet (a), FSR 2 on the posterior facet, FSR 3 and 5 on the lateral facet (b) and FSR 4 on the medial facet. FSR 6 was bonded under the collar of the stem. Note that a plastic adhesive enfolded the stem and the FSR to guarantee impermeability and permanent electrical continuity (c).

harvesting was 94.25 days [Range, 63-119]. The specimens were stored at $4{ }^{\circ} \mathrm{C}$ before harvesting. Preoperative planning was performed with stereoradiography system EOS (EOS - Imaging Paris) on antero-posterior (AP) and lateral views. A cementless collared revision femoral stem coated with hydroxyapatite was investigated (Kar, DePuy France).

The insertion procedure was performed according to the protocol described for this type of stem. A transversal diaphyseal osteotomy was performed at $25 \mathrm{~cm}$ below the lesser trochanter. The femurs were then potted in a steel box at $11^{\circ}$ adduction and $4^{\circ}$ of flexion (Fig. 5). This configuration was validated with a preliminary testing of two intact femurs harvested from one female and one male cadaver ( 81 and 82 years old). The purpose of this preliminary testing was to evaluate the fracture location under a destructive testing to confirm if clinically relevant failure modes could be obtained. The load was applied to the femoral head. The same intracapsular base neck fracture localization was obtained for both of the femurs. These fractures were similar to spontaneous fracture of the hip due to osteoporosis and correspond to the maximum stress in finite elements models studies [14]. The failure loads found in this study $7332 \mathrm{~N}$ [Range, 5843-8822] were comparable to the values reported in the literature [14,15]. We

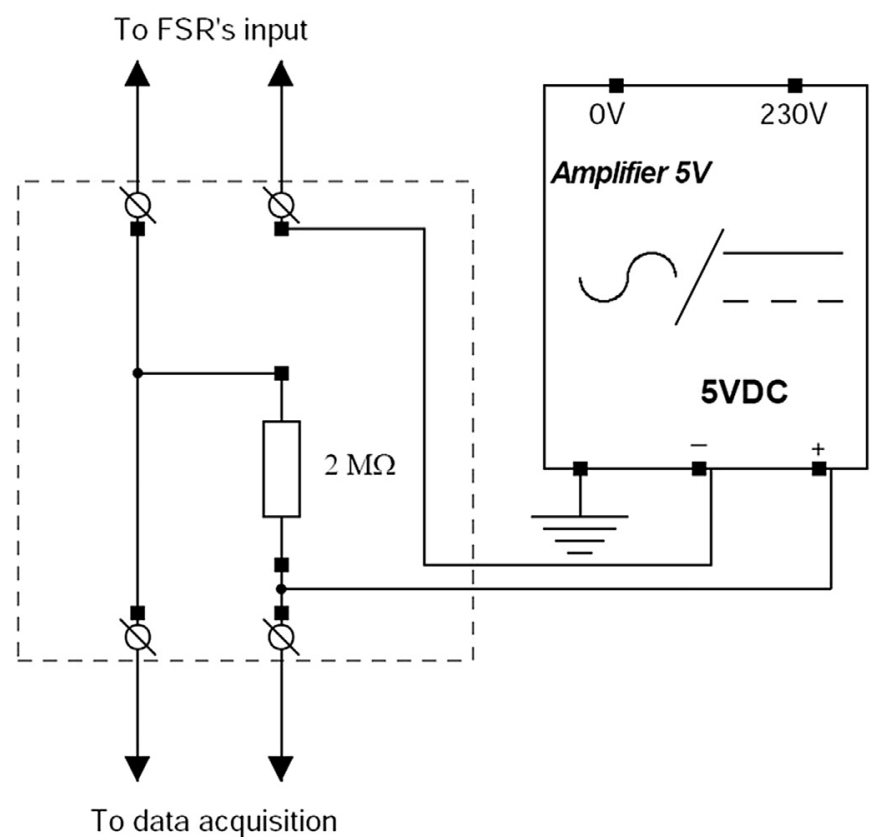

Fig. 4. Signal conditioning circuit.

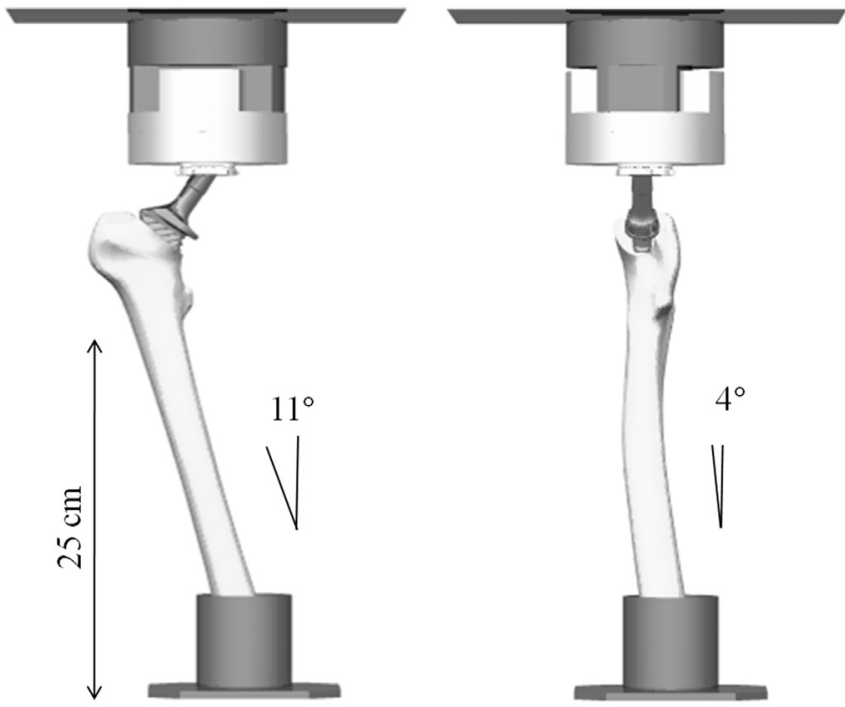

Fig. 5. Experimental setup. The femurs were potted in a steel box at $11^{\circ}$ adduction and $4^{\circ}$ of flexion according to the literature data.

sustained in regard to these findings that our experimental protocol could be exploited.

\subsection{Tests on implanted femurs}

Destructive tests were performed on the eight implanted femurs with a loading machine (5500-R, Instron, Canton, MA, USA) at a constant displacement rate of $10 \mathrm{~mm} / \mathrm{min}$. Load and displacement were recorded via a load cell (Instron Load Cell $100 \mathrm{kN}$ Static, Cat No 2518-201, Code 100, Instron, Canton, MA, USA) by the testing machine. The load was applied to the stem through a movable hip socket. Muscle forces were not simulated. During the destructive test, the event was filmed by means of two numeric cameras (Canon, Digital Video CamCorder MV901). FSR pressure values 
were recorded. The calibration process was performed on one FSR after retrieval of the stem in order to assess the viability and accuracy of the measurements of the FSR. The curve obtained is showed in Fig. 6. This control was not performed for each FSR. All experiments were made at room temperature ranging from 22 to $25^{\circ} \mathrm{C}$.

\section{Results}

\subsection{Failure mode}

The average failure load was 4241.25 N [Range, 2665-6442] and the average failure displacement was $17.54 \mathrm{~mm}$ [Range, 10-27]. Failure loads and displacement for each specimen are recorded in Table 1.

\subsection{FSR data}

FSR maximum contact pressure values are recorded in Table 2. The maximum contact pressure was measured for FSR 4 and averaged 1.965 MPa [Range, 0.075-5.247]. FSR 6 were loaded when the implant collar-calcar contact was achieved, which was clinically and radiologically observed in five cases. FSR 3 was damaged after implantation in cases 2, 3 and 8.

\section{Discussion}

Our aim was to gather in vitro pressure values at the implant-bone interface after implantation of a cementless femoral stem in realistic conditions. Interfacial pressure values could be obtained for the eight cases despite three damaged sensors. This experimental setup is innovative and opens a novel field of in vitro investigations of cementless implants.

The previously published literature does not provide much data on implant-bone load transfer for cementless implants. Values

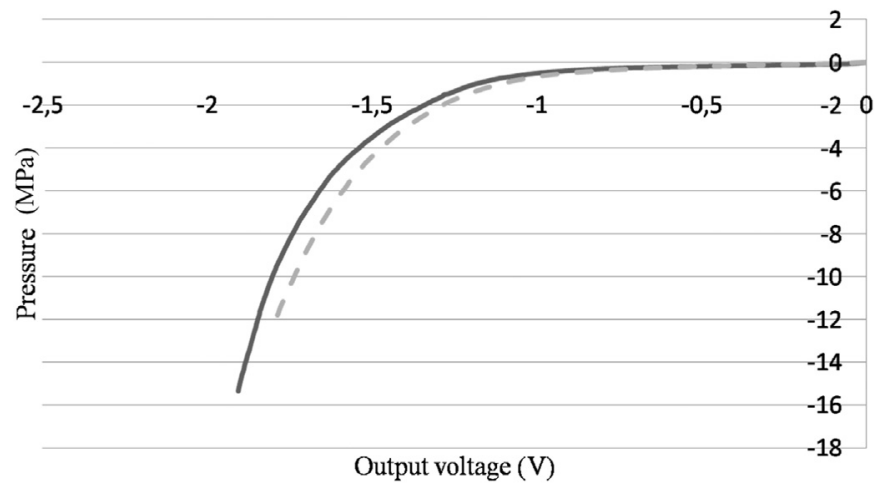

Fig. 6. Comparison between the calibration curve of the FSR before the experimentation (black line) and the calibration curve after the stem retrieval (dashed line).
Table 2

Values of maximum pressure contact for each FSR are reported (MPa). It is mentioned whether collar-calcar contact is achieved during the test. Underlined values indicate the damaged FSR sensors.

\begin{tabular}{llllllll}
\hline Specimen & FSR 1 & FSR 2 & FSR 3 & FSR 4 & FSR 5 & FSR 6 & $\begin{array}{l}\text { Collar-calcar } \\
\text { contact }\end{array}$ \\
\hline 1 & & & & & & & Fes \\
2 & 0.074 & 0.082 & 0.941 & 5.247 & 0.081 & 0.022 & Yes \\
3 & 0.016 & 0.023 & $\underline{0.000}$ & 0.272 & 0.000 & 0.206 & No \\
4 & 0.066 & 0.068 & $\underline{0.000}$ & 0.094 & 0.045 & 0.039 & No \\
5 & 0.108 & 0.089 & 0.072 & 3.294 & 0.081 & 0.227 & Yes \\
6 & 0.011 & 0.061 & 0.118 & 2.916 & 0.000 & 0.013 & No \\
7 & 0.000 & 0.037 & 0.080 & 0.075 & 0.049 & 0.22 & Yes \\
8 & 0.011 & 0.013 & 3.141 & 0.390 & 0.038 & 0.21 & Yes \\
\hline
\end{tabular}

available are derived from strain measurements provided by strain gauges bonded on the external cortex of the femur. The work of Sakai et al. remains the only reference comparable to our study [16]. They have used pressure films and tactile sensors to measure contact pressure at the implant-bone interface. Synthetic femurs were bisected and subsequently fixed using screws after implantation of the instrumented femoral stem. The maximum pressure values were higher in Sakai's work [Range 0 - over $25 \mathrm{MPa}$ ], which may rely on the femur stiffness modification. But comparing pressure distribution is uneasy, taking into account that pressure measurements were limited to each FSR active area in our study. Therefore, an extensive cartography of contact pressure distribution within the implant-bone interface could not be obtained. Using custom built FSR with a wider active area or increasing their number would have provided more pertinent data like pressure films used by Sakai et al. [16].

Piezoelectric sensors were also investigated with good results in cemented but not for cementless devices. Like FSR sensors, piezoelectric sensors need contact application at the exact point of sensor location [13]. Prediction of implant-bone contacts using the preoperative planning would have allowed a more relevant positioning of the sensors in this study. However, a standardized positioning was preferred in this preliminary work to assess FSR comportment throughout implantation. FSR number and locations were selected arbitrary, but similar in each case, for convenient bonding conditions.

FSR sensors have a large footprint but their force sensitivity is optimized for use in human touch. They have been used in clinical evaluation of hand function and also lower limbs amputees for assessments of pressure at the stump/prosthetic socket interface in lower limb amputees $[17,18]$. This current study reports the first exploitation in joint replacement implants biomechanics. FSR reached the geometrical and structural properties required for our investigation. However, as interfacial pressure values were poorly available in the literature, the FSR ability for recording the range of pressure values in this application was unpredictable. The maximum pressure values obtained ( $5 \mathrm{MPa}$ ) were in the pressure sensitivity range of the FSR (0.05 MPa to over $5 \mathrm{MPa})$. Moreover, the

Table 1

Clinical data. Failure load and displacement are noted for each specimen.

\begin{tabular}{|c|c|c|c|c|c|}
\hline Specimen & Gender & Age (years) & Implant size (mm) & Failure displacement (mm) & Failure load $(\mathrm{N})$ \\
\hline 1 & $\mathrm{H}$ & 89 & 14 & 6 & 3748 \\
\hline 2 & $\mathrm{H}$ & 87 & 12 & 10 & 3784 \\
\hline 3 & $\mathrm{H}$ & 74 & 12 & 13 & 2665 \\
\hline 4 & $\mathrm{~F}$ & 86 & 12 & 23 & 3526 \\
\hline 5 & $\mathrm{~F}$ & 51 & 14 & 30 & 3523 \\
\hline 6 & $\mathrm{H}$ & 76 & 12 & 23 & 5828 \\
\hline 7 & $\mathrm{H}$ & 88 & 16 & 11 & 2723 \\
\hline 8 & $\mathrm{H}$ & 91 & 12 & 23 & 5482 \\
\hline
\end{tabular}


calibration was performed for pressure values reaching $15 \mathrm{MPa}$. Even though the previous experimental use of FSR was for lower range of pressure values, FSR allow accurate pressure measurements in the higher dynamic range of pressure. We emphasize the fact that FSR sensors only measure the normal component of the force (compressive force) applied at the bone-implant interface. The values of the pressure measured with the FSR and reported here do not reflect the whole generated stress at the implant-bone interface. During the destructive tests performed in this study the shear force component was probably not negligible but not measured by the FSR. However, the main idea of this experimental study was more to cartography the pressure distribution at the implant-bone interface than to obtain true accurate measurements of the pressure values. The further steps of this study is to develop a finite element model based on this experimentation and compare the numerical model and the in vitro experimentation in terms of pressure values and bone-implant contact topography.

The two major limitations of using FSR for measuring compression force in biomechanics application are sensitivity to shear forces and hysteresis. Shear forces may indeed affect the sensitivity of FSR sensors. As we said above, the pressures values measured in this study only reflect an approximation of the pressure values of the normal stress component at the bone-implant interface. A technique for optimizing FSR calibration as been described, by use of incorporating terms dependent on the past loading history in the calibration equation to compensate hysteresis and by use of prolonged preloading of the FSR in compression and shear to eliminate sensitivity to shear loading [19]. This optimization is relevant for cyclic tests and was not performed in this destructive tests study.

The mean compressive destructive load reached $4000 \mathrm{~N}$ during the tests, which may have generated excessive shear forces within implant-bone interface and subsequently delamination of the sensors' layers. Three FSR were damaged but near the soldered tabs but not by delamination as we could observed after implant retrieval. The other FSR were all in apparent working condition. Moreover, the calibration curve obtained for one FSR after retrieval of the stem was similar as the index curve (Fig. 6).

Interfacial contact that occurs between stem and bone involve an irregular surface of engagement, with local asperities, which can cause false triggering of the sensors. Moreover, applying FSR to curved surfaces may cause pre-loading of the device as the two opposed layers are forced into contact by the bending tension. The degree of curvature over which an FSR can be bent is a function of the size of the active area. The smaller the active area, the less effect a given curvature will have on the FSR response. A zero setting was performed before implantation to annul any substantial preloading of the device. But involvement of the bending tension in the pressure measurements after implantation is difficult.

Based upon these data, some clinical observations can be made. Proximal contact-free areas on radiographs at the level of FSR 1, 2 and 5 were poorly loaded throughout the test. FSR 3 and 4 were progressively loaded when subsidence of the stem occurred (Fig. 7). The damaged FSR location (FSR 3) suggests that high friction areas were on lateral aspect of the stem. The measurements confirmed the absence of load transfer in the proximal femur which is at origin of stress shielding which is involved in bone resorption of the proximal femur $[1,20]$. This supports clinical findings of stress shielding after revision surgery with cementless stems [21-25].

A wide range of contact pressure values was measured within the same femur at the same loading phase. These values were obtained experimentally throughout destructive loading tests that did not represent clinical situations. They however emphasized the variability of pressure amplitude and distribution within the implant-femur interface, which supports the experimental findings of Sakai et al. [16].

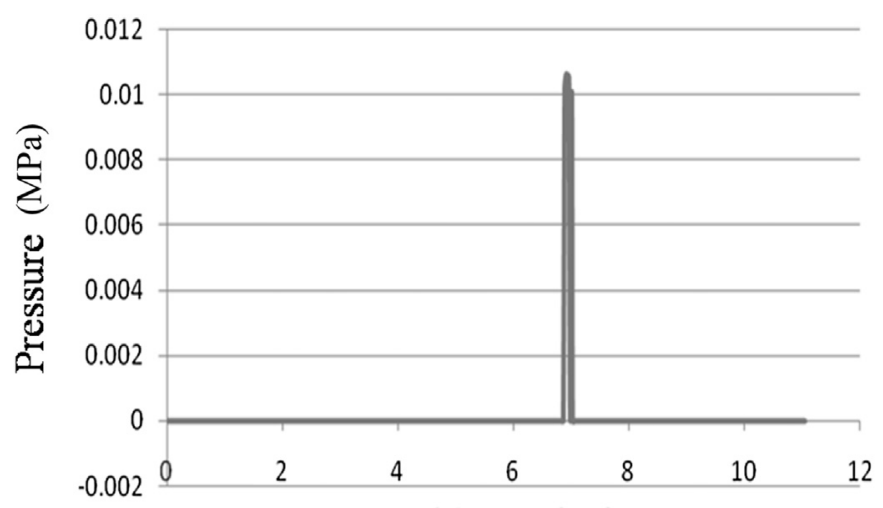

\section{Displacement (mm)}

Fig. 7. Response of FSR 3 throughout loading. The sensor was progressively loaded and pressure values could be recorded (maximum $3 \mathrm{MPa}$ ).

This experimentation provided quantitative data on contact pressure between cementless stems and cadaver femurs. We achieved measurements within the implant-femur interface without damaging the femur, which provided realistic conditions. Such measurements could be of valuable interest for finite element models validation. FSR reached many of the requirements for an ideal implant-bone interfacial sensor.

\section{Conflict of interest}

The authors declare that they have no conflict of interest.

\section{References}

[1] Huiskes R, Weinans $H$, van Rietbergen B. The relationship between stress shielding and bone resorption around total hip stems and the effects of flexible materials. Clin Orthop Relat Res 1992 Jan;274:124-34.

[2] Viceconti M, Brusi G, Pancanti A, Cristofolini L. Primary stability of an anatomical cementless hip stem: a statistical analysis. J Biomech 2006;39(7): 1169-79.

[3] Viceconti M, Pancanti A, Dotti M, Traina F, Cristofolini L. Effect of the initial implant fitting on the predicted secondary stability of a cementless stem. Med Biol Eng Comput 2004 Mar;42(2):222-9.

[4] Lengsfeld M, Burchard R, Gunther D, Pressel T, Schmitt J, Leppek R, Griss P. Femoral strain changes after total hip arthroplasty - patient-specific finite element analyses 12 years after operation. Med Eng Phys 2005 Oct;27(8): 649-54.

[5] Joshi MG, Advani SG, Miller F, Santare MH. Analysis of a femoral hip prosthesis designed to reduce stress shielding. J Biomech 2000 Dec;33(12):1655-62.

[6] Sumner DR. Long-term implant fixation and stress-shielding in total hip replacement. J Biomech 2015 Mar 18;48(5):797-800.

[7] Caouette C, Yahia LH, Bureau MN. Reduced stress shielding with limited micromotions using a carbon fibre composite biomimetic hip stem: a finite element model. Proc Inst Mech Eng H 2011 Sep;225(9):907-19.

[8] Boyle C, Kim IY. Comparison of different hip prosthesis shapes considering micro-level bone remodeling and stress-shielding criteria using threedimensional design space topology optimization. J Biomech 2011 Jun $3 ; 44(9): 1722-8$.

[9] Yamako G, Chosa E, Totoribe K, Hanada S, Masahashi N, Yamada N, Itoi E. Invitro biomechanical evaluation of stress shielding and initial stability of a lowmodulus hip stem made of beta type Ti-33.6Nb-4Sn alloy. Med Eng Phys 2014 Dec;36(12):1665-71.

[10] Piao C, Wu D, Luo M, Ma H. Stress shielding effects of two prosthetic groups after total hip joint simulation replacement. J Orthop Surg Res 2014;9:71.

[11] Bieger R, Ignatius A, Reichel H, Durselen L. Biomechanics of a short stem: in vitro primary stability and stress shielding of a conservative cementless hip stem. J Orthop Res 2013 Aug;31(8):1180-6.

[12] Cristofolini L, Juszczyk M, Taddei F, Field RE, Rushton N, Viceconti M. Stress shielding and stress concentration of contemporary epiphyseal hip prostheses. Proc Inst Mech Eng H 2009 Jan;223(1):27-44.

[13] Cristofolini L, Marchetti A, Cappello A, Viceconti M. A novel transducer for the measurement of cement-prosthesis interface forces in cemented orthopaedic devices. Med Eng Phys 2000 Sep;22(7):493-501. 
[14] Duchemin L, Mitton D, Jolivet E, Bousson V, Laredo JD, Skalli W. An anatomical subject-specific FE-model for hip fracture load prediction. Comput Methods Biomech Biomed Eng 2008 Apr;11(2):105-11.

[15] Cristofolini L, Juszczyk M, Martelli S, Taddei F, Viceconti M. In vitro replication of spontaneous fractures of the proximal human femur. J Biomech 2007:40(13):2837-45.

[16] Sakai R, Itoman M, Mabuchi K. Assessments of different kinds of stems by experiments and FEM analysis: appropriate stress distribution on a hip prosthesis. Clin Biomech (Bristol, Avon) 2006 Oct;21(8):826-33.

[17] Nikonovas A, Harrison AJ, Hoult S, Sammut D. The application of force-sensing resistor sensors for measuring forces developed by the human hand. Proc Inst Mech Eng H 2004;218(2):121-6.

[18] Buis AW, Convery P. Calibration problems encountered while monitoring stump/socket interface pressures with force sensing resistors: techniques adopted to minimise inaccuracies. Prosthet Orthot Int 1997 Dec;21(3): $179-82$.

[19] Hall RS, Desmoulin GT, Milner TE. A technique for conditioning and calibrating force-sensing resistors for repeatable and reliable measurement of compressive force. J Biomech 2008 Dec 5;41(16):3492-5.
[20] Decking R, Puhl W, Simon U, Claes LE. Changes in strain distribution of loaded proximal femora caused by different types of cementless femoral stems. Clin Biomech (Bristol, Avon) 2006 Jun;21(5):495-501.

[21] Moreland JR, Moreno MA. Cementless femoral revision arthroplasty of the hip: minimum 5 years followup. Clin Orthop Relat Res 2001 Dec;393: 194-201.

[22] Bohm P, Bischel O. The use of tapered stems for femoral revision surgery. Clin Orthop Relat Res 2004 Mar;420:148-59.

[23] Weeden SH, Paprosky WG. Minimal 11-year follow-up of extensively porouscoated stems in femoral revision total hip arthroplasty. J Arthroplasty 2002 Jun;17(4 Suppl. 1):134-7.

[24] Crawford CH, Malkani AL, Incavo SJ, Morris HB, Krupp RJ, Baker D. Femora component revision using an extensively hydroxyapatite-coated stem. J Arthroplasty 2004 Jan;19(1):8-13.

[25] Nishino T, Mishima H, Kawamura H, Shimizu Y, Miyakawa S, Ochiai N. Followup results of 10-12 years after total hip arthroplasty using cementless tapered stem - frequency of severe stress shielding with synergy stem in Japanese patients. J Arthroplasty 2013 Dec;28(10):1736-40. 